\title{
A clinical librarian pilot project in psychiatry ${ }^{\dagger}$
}

\author{
Helene Gorring, ${ }^{1}$ Erin Turner, ${ }^{2}$ Ed Day, ${ }^{2}$ Christopher A. Vassilas, ${ }^{3}$ Mark Aynsley ${ }^{4}$
}

The Psychiatrist (2010), 34, 65-68, doi: 10.1192/pb.bp.108.024364

${ }^{1}$ Birmingham \& Solihull Mental Health Foundation Trust; ${ }^{2}$ Slade Road Centre, Erdington, Birmingham; ${ }^{3}$ Ashcroft Unit, The Moorings, Birmingham; ${ }^{4}$ The Barberry, Edgbaston, Birmingham Correspondence to Christopher A. Vassilas (c.a.vassilas@bham.ac.uk)
Aims and method A 4-month pilot was conducted to assess whether the clinical librarian model, which has been successfully used within acute hospitals, would work in a mental health setting. A librarian attended weekly clinical team meetings in two community mental health teams to help generate clinical questions. A summary of the evidence on each topic was then presented the following week. An evaluation of the pilot was carried out using a questionnaire survey, focus groups and interviews.

Results Results suggest that the project had produced a positive impact within the teams and begun the process of embedding evidence-based information within clinical practice.

Clinical implications With some adaptations, the clinical librarian model can be an effective method of implementing evidence-based practice and addressing continuing professional development needs within mental health clinical teams.

Declaration of interest None.
The drive towards evidence-based healthcare has been enshrined in recent UK government policy, and the ability to search, appraise and apply research evidence has become a standard part of medical training. Sackett et $a l^{1}$ have advocated the 'conscientious, explicit, and judicious use of current best evidence in making decisions about the care of individual patients... [by] using the best available external evidence'. However, it is clear that health professionals do not always search the literature systematically when faced with a clinical question, and often prefer to ask a colleague or fail to follow it up altogether. ${ }^{2}$ Lack of time and limited skills in literature searching are often cited as the main reasons for this, and Sackett et al have championed the idea of a clinical librarian who is present in clinical settings such as team meetings or ward rounds. By being readily accessible when the clinical questions arise, the librarian can act as a fast and efficient route to the best available evidence. The clinical librarian model has been promoted by a number of acute hospital trusts in the UK, and the Commission for Health Improvement report on University Hospitals Leicester National Health Service (NHS) Trust in 2002 concluded that other NHS organisations could learn from their clinical librarian service. ${ }^{3}$ No evidence was found of mental health organisations using this approach. This paper describes a 4-month pilot conducted in Birmingham and Solihull Mental Health Foundation Trust (BSMHFT).

\section{Method}

Birmingham \& Solihull Mental Health Foundation Trust provides mental healthcare for the city of Birmingham. In

†See commentary, pp. 68-70, this issue. late 2007, the BSMHFT library committee approved a trial of a clinical librarian visiting two clinical teams once per week over a 4-month period. The two teams nominated to take part were the Slade Road Community Drug Team and Mental Health Services for Older People's Edgbaston Community Team. At the time of the pilot, each team consisted of nine members, with a consultant psychiatrist and specialist registrar as part of the team; other team members were from nursing, counselling, psychology and social work backgrounds.

A librarian (M.A.) attended the weekly multidisciplinary team meetings with the aim of assisting the staff members in answering clinical questions that might arise during case discussions. He also had a brief to work with the consultant psychiatrist in each team (E.D. and C.A.V.) to assist team members in setting more focused clinical questions, and in appraising any evidence produced. Each week clinical questions were set by the team and the librarian took them back to the library at the end of the meeting. During the following week he undertook a comprehensive search of bibliographic databases including Cochrane, MEDLINE and Embase and produced a brief (500-1000 words) summary of the evidence available to present at the next meeting. Where appropriate, he also found copies of the full text of selected clinical papers. The team discussed the findings at their next meeting, and action points were recorded where relevant.

The pilot was evaluated using a questionnaire containing five open-ended questions presented to all members of both clinical teams at the end of the 4-month period. Responses were then followed up with a focus group conducted by the lead librarian (H.G.) in each team. Interviews were also carried out by one of the authors with both consultant leads, as well as with the librarian. 
Open-ended questions were used throughout, and staff were asked about the impact that the librarian's attendance had on their meetings, the utility of any evidence produced, the processes of communication between the clinical team and librarian, and the overall impact on clinical practice. A simple thematic analysis was conducted to draw out the main findings.

\section{Results}

The overall response rate for the questionnaire was $78 \%$ (14/18). Over the 4-month period a total of eight searches were conducted for the old age psychiatry team and nine for the community drug team. A limited number of searches yielded 'high-level evidence' in the form of systematic reviews or good-quality randomised controlled trials (Table 1). A range of other materials was produced, including clinical guidelines and chapters from textbooks.

The following key themes were drawn from the clinicians' responses, and illustrated by direct quotations where relevant.

\section{Impact on evidence-based practice}

Team members in both teams commented that they felt that the project had resulted in positive changes to their clinical practice, and a number of specific examples were given. The specialist registrar from the old age team reported that the material supplied at one meeting assisted him in providing better information to a patient and their family. Threequarters (6/8) of the respondents in the community drug team specifically mentioned the impact of evidence presented on motivational interviewing which had resulted from a case presentation of a patient who had dropped out of treatment. Two typical quotes were:
The papers and articles we have looked at on motivational interviewing have influenced my style of practice, and have also made me question the style I was previously adopting. (Substance misuse clinician)

These changes are difficult to pinpoint but there is noted change in emphasis and a greater willingness to embrace things like motivational enhancement techniques. (Team manager)

\section{More than a literature search service}

Much of the feedback suggested that there was value in a librarian attending clinical team meetings that could not have been achieved by the library's existing literature search service alone. The librarian felt that attending the meetings had enabled him to build up some expertise in new clinical areas, and provided important contextual information on how a clinical question had arisen. Both consultant psychiatrists highlighted the benefit of an external person from a different professional background attending the team meeting, and this point was reiterated by several of the team members. Reference was made to the librarian's 'unbiased, unemotional and informed contributions to meetings', and the fact that his presence forced teams to consider the clinical evidence that might inform a decision. As one of the junior doctors commented, 'in other teams these questions are raised but not discussed or followed up'.

\section{A tool to assist continuing professional development}

Both doctors and other clinicians reported that the librarian's input had been a useful training tool. Team members from both teams felt that the setting and answering of clinical questions 'broadened the knowledge base', and that the process led to a better understanding of 'the librarian's potential role in enhancing learning'. Having a clinical librarian present facilitated the development of a

\section{Table 1 A selection of questions raised by the clinical teams and the evidence found}

Question

1. Do opiate users have different predictive risk factors for suicide than the general population?
Evidence found

Both topics have been researched independently but few review articles compare the two groups. A 2002 paper concluded that the major risk factors apply to both, but that heroin users have a far wider exposure to these factors and are 14 times more likely to die from suicide than their peers indicating the need for routine screening.

2. Are psychosocial treatments combined with agonist maintenance NICE guideline

treatments better than agonist treatments alone for the treatment of opioid dependence?

Heath Technology Assessment

A Cochrane review looked specifically at this question.

3. Is motivational interviewing more effective than standard care/ There is good quality evidence in this area. An RCT published in counselling in engaging individuals who have been opiate dependent 1995 is still being cited as a key type 1 evidence paper by the for at least 3 months?

4. What is the evidence for the use of antipsychotics or antidepressants as an intervention for insomnia in individuals with dementia? National Treatment Agency for Substance Misuse.

A review concluded that although widely used, the effectiveness of pharmacological agents has not been demonstrated in controlled trials with Alzheimer's disease. A planned Cochrane review on pharmacological interventions for dementia was abandoned due to lack of progress.

5. Is cognitive training an effective intervention for memory impairment in dementia?

NICE and a Cochrane review concluded that there was insufficient evidence to recommend this and that more research is needed. Another meta-analysis with wider inclusion criteria was more optimistic. 
strategy for addressing knowledge gaps. Clinicians from both teams began to ask more questions as the project progressed, and to take more interest in the answers. The curiosity that this process generated was reflected by respondents from the community drug team who subsequently booked onto library training courses and were keen that training on literature searching be organised as part of a future team meeting. One team member commented that 'having a librarian attend meetings helps to break down barriers to visiting the local library, whether it is from lack of confidence, or simply because they [clinicians] do not see it as relevant'. The specialist registrar also reflected that she thought it was useful for the librarian to attend for a set period, to 'generate interest in clinical questioning and model some good literature searches for long enough to change behaviours, i.e. inspire clinicians to do the same, but not long enough to create dependency or laziness'.

Although respondents admitted that the clinical librarian service had been beneficial for evidence-based practice and that the succinct summaries were very useful, it was recognised in the interviews with both the consultant leads and the librarian that the role clearly needed the support of a consultant or specialist registrar leading the team meeting to facilitate the generation of clinical questions.

\section{Other key benefits highlighted}

Respondents from both teams commented on the benefit of having timely access to the information they needed, 'fast turnaround' being cited as one of the key benefits in both teams. Birmingham \& Solihull Mental Health Foundation Trust is a large, geographically dispersed organisation and there was an appreciation of the library 'outreach' element of this service, this being a 'most efficient way of bringing evidence-based practice to the centre' (substance misuse clinician), and an 'in-house platform to put across clinical questions' (specialist registrar, old age psychiatry).

In addition, many of the participants in the community drug team identified clinical question formulation as one of the main benefits of the pilot. One commented that 'as a team we have become better at framing clinical questions; putting them together to be able to get the answer or information we have been after'. The interview with the librarian corroborated that where discussions had been held to generate, clarify and reach a consensus around a clinical question, this had been an effective way of providing multidisciplinary involvement.

\section{Discussion}

This was a small pilot study, but the results suggest that the clinical librarian paradigm can be usefully applied in mental health services. However, differences between psychiatry and Sackett et al's ${ }^{1}$ acute medical care model should be noted. Having taught staff about the importance of a hierarchy of evidence, it is important to ensure that a lack of 'high-level' evidence from meta-analyses or randomised controlled trials in the psychiatric field does not become demotivating for clinical staff. The team should be encouraged to use the best available evidence, and examples were cited by the librarian whereby information from a good textbook had been more appropriate, for example a question about interview techniques for suicide assessment in the community drug team and a query about the cut-off point for the semantic fluency test in dementia in the old age psychiatry team.

\section{Extending the programme}

Following this pilot, BSMHFT has agreed to extend the clinical librarian programme to other teams, with some modifications. First, there was a general consensus that weekly attendance was too frequent. The model of using team discussion to generate, clarify, and reach a consensus around a clinical question seemed to be an effective method to ensure involvement of all team members.

The role of clinical librarian requires a dedicated librarian with experience in advanced literature searching and with some critical appraisal skills. However, the literature supports the premise that this use of relatively expensive librarians' time is cost-effective. Urquhart et al calculated that the cost of employing a clinical librarian to carry out searches in a clinical team in north Wales led to an overall cost saving to the team of $£ 136$ per week, based on the premise that cost of the librarian carrying out searches was less than that of clinicians and that searches clinicians carried out were more efficient following the introduction of a clinical librarian. Since it is clearly impractical for this service to be provided to all 130 teams within the Trust for a prolonged period, it has been decided that a librarian will attend a team meeting for a set period of a few months to generate interest in clinical questioning and literature searching, before moving on to a different team. The frequency of attendance will be monthly.

The role of the clinical librarian is consistent with the New Ways of Working agenda. ${ }^{4}$ However, it was felt to be important that a senior medical or nursing lead was present to help facilitate the discussion and the formulation of questions. This person also needed to take responsibility for conducting preliminary work with the team about the principles of evidence-based medicine, and modelling the framing of clinical questions and the application of evidence to clinical practice. The service will only be offered to teams where a senior clinician willing to take on this role can be identified.

The last word is best left to one of the participating team members:

It has been great to be part of this and I hope it continues. It has stimulated good team discussions around issues and ways of working. Team meetings seem to have a sense of purpose of sharing experiences, research, keeping up to date, improving knowledge, and improving relationships and dynamics of team group.

\section{About the authors}

Helene Gorring is Head of Library Services at Birmingham \& Solihull Mental Health Foundation Trust, Erin Turner is Specialist Registrar in Psychiatry and Ed Day is Consultant Psychiatrist, Slade Road Centre, Christopher A.Vassilas is Consultant Psychiatrist at the Ashcroft Unit, and Mark Aynsley is Clinical Librarian at The Barberry, all services based in Birmingham. 


\section{References}

1 Sackett DL, Rosenberg WMC, Muir Gray JA, Brian Haynes RB, Scott Richardson W. Evidence based medicine: what it is and what it isn't. BMJ 1996; 312: 71-2

2 Davidoff $F$, Florance $V$. The informationist: a new health profession? Ann Intern Med 2000; 132: 996-8.

3 Booth A, Sutton A, Falzon L. Evaluation of the Clinical Librarian Project:
University Hospitals of Leicester NHS Trust. School of Health and Related Research, University of Sheffield, 2002.

4 Care Services Improvement Partnership, National Institute for Mental Health in England. New Ways of Working for Everyone. Department of Health, 2007.

5 Urquhart C, Durbin J, Turner J. North Wales Clinical Librarian Project: Final Project Report. Department of Information Studies, University of Wales Aberystwyth, 2005 (http://hdl.handle.net/2160/195).

\title{
COMMENTARY
}

\section{Preparing for practice: a higher education perspective. Commentary on ... A clinical librarian pilot project in psychiatry ${ }^{\dagger}$}

\author{
Pat Spoor, ${ }^{1}$ Liz Neilly ${ }^{1}$
}

The Psychiatrist (2010), 34, 68-70, doi: 10.1192/pb.bp.109.027441

${ }^{1}$ Health Sciences Library, University of Leeds

Correspondence to Liz Neilly (e.m.neilly@leeds.ac.uk)
Summary Research suggests that contact time between clinical team and clinical librarian can have a direct and positive impact on patient care through encouraging a more rigorous approach to information retrieval and appraisal of the evidence base. This commentary focuses on the findings of a specific clinical librarian pilot project in a mental health trust. It discusses how clinicians could be better supported to develop improved information literacy skills through initiatives in higher education and what impact this might have on the model of clinical librarianship proposed by the project.

\section{Declaration of interest None.}

\section{Clinical librarians in England}

The concept of the clinical librarian working closely with clinical teams to support evidence-based practice has been around for about 30 years. Currently, there are around 50 clinical librarians in England, ${ }^{1}$ mainly based in the acute sector. ${ }^{2}$ Although it is difficult to quantify, and most of the evaluations of the model undertaken are based on studies that are not methodologically robust, there is some evidence of the positive effect of clinical librarianship on patient care. $^{3-5}$ Numbers of clinical librarians are growing, but scalability is an issue. The Hill review ${ }^{1}$ states that it is unrealistic to expect the widespread implementation of such a policy', recommending instead that resources be focused on key areas within acute hospitals where the impact on patient care might be maximised.

'See education \& training, pp. 65-68, this issue.
In contrast to Hill's view, such characteristics of mental health services as geographical dispersion of services, problems with staff access to IT, and a paucity of highquality evidence, make experimenting with the clinical librarian model in this setting worthwhile, and there is some indication that these very factors mean outreach library services can make a positive contribution to clinical practice. $^{6}$

\section{The Birmingham pilot service}

Gorring et $a l^{2}$ have highlighted the positive impact that the clinical librarian may have as a catalyst stimulating the clinical team to form answerable clinical questions and think about how they might respond using the best available evidence to improve patient care. The extension of the project attempts to address the 